\title{
Dirac zero-modes in compact $U(1)$ gauge theory
}

\author{
Thomas Drescher and C.B. Lang \\ Institut für Physik (Theoretische Physik), \\ Universität Graz, A-8010 Graz, AUSTRIA
}

February 18, 2019

\begin{abstract}
We study properties of the zero and near-zero eigenmodes of the overlap Dirac operator in compact $U(1)$ gauge theory. In the confinement phase the exact zero-modes are localized as found by studying the values of the inverse participation ratio and other features. Nonzero-eigenmodes are less localized in the confinement phase. In the Coulomb phase no zero-modes are observed and the eigenmodes show no localization at all.
\end{abstract}

PACS: 11.15.Ha

Key words: Lattice field theory, Dirac operator spectrum, topology 


\section{Motivation}

The Atiyah-Singer index theorem [1] relates the topological charge of the background gauge field configuration to the number of fermionic zero-modes of the Dirac operator. The theorem states a particular connection between the background gauge field and the fermionic fields. It is a theorem derived on differentiable manifolds, however. Quantum field configurations and in particular lattice fields are non-differentiable. Hence it is of interest to study on the lattice the topology of the gauge background as well as the properties of the zero-modes of the Dirac operator.

A major progress in understanding the manifestation of the index theorem on the lattice was the realization that the eigenvectors of a $\gamma_{5}$-hermitian lattice Dirac operator with real eigenvalues should be interpreted as the lattice counterparts of the continuum zero-modes. This can be understood from the fact that only eigenvectors $\psi$ with real eigenvalues can have non-vanishing pseudoscalar matrix elements $\left\langle\psi\left|\gamma_{5}\right| \psi\right\rangle$, like the zero-modes in the continuum.

Continuum QED in 4D in its usual non-compact realization for the gauge field has no topological charge. For the lattice formulation with compact representation of the gauge fields on the other hand the situation changes. Lattice QED exhibits a twofold phase structure: a physical one, containing the massless photon and a confining phase with properties similar to QCD. Some phenomena appearing in the confinement phase are: zero modes, magnetic monopole-antimonopole pairs and the occurrence of a non-zero chiral condensate.

Chiral symmetry breaking is a key feature of the theory of strong interactions, QCD. The order parameter is the chiral condensate $\langle\bar{\psi} \psi\rangle$; it is related to the density of eigenvalues $\rho(\lambda)$ of the Dirac operator near the origin via the Banks-Casher relation [2]. As compact lattice QED shows a confining phase for certain values of the coupling constant one also expects [3] and indeed observes [3] the appearance of a chiral condensate.

For a study of zero-modes and chiral symmetry breaking it is advisable to work with chirally symmetric Dirac operators. On the lattice chiral symmetry is realizable in a locally violated form, expressed through the GinspargWilson relation [4] which in its original form reads:

$$
\gamma_{5} \mathcal{D}+\mathcal{D} \gamma_{5}=2 a \mathcal{D} \gamma_{5} R \mathcal{D}
$$

where $\mathcal{D}$ denotes the massless Dirac operator, $a$ is the lattice spacing and $R$ a local function of the gauge fields. In the continuum limit $\mathcal{D}$ anticom- 
mutes with $\gamma_{5}$ thus showing chiral symmetry. Dirac operators satisfying the Ginsparg-Wilson relation preserve a lattice version of chiral symmetry [5] without fermion doubling. For a given local operator $R$ there may be many Dirac operators. The eigenvalue spectrum of a Dirac operator for $R=\frac{1}{2}$ lies on a unit circle around the center $1 / a$ and complex eigenvalues come in complex conjugate pairs. Only for real $\lambda$ the expectation value of $\gamma_{5}$ between the eigenstates $\left\langle\psi_{\lambda}\left|\gamma_{5}\right| \psi_{\lambda}\right\rangle$ is non-zero. Therefore exact zero-modes have definite chirality.

Although there are several realizations of the Dirac operator satisfying the Ginsparg-Wilson relation approximately, only the numerically costly overlap operator [6] is an exact realization. Within QCD there have been several studies on the density of near-zero-modes and the properties of exact zeromodes and their relationship to topological excitations (see e.g. [7, 8, 9, 10]).

In compact lattice QED there have been studies with the overlap operator 11] demonstrating the existence of zero-modes in the confined phase and relating the density of the near-zero-modes to the universal distributions from Random Matrix Theory. Here we want to continue the study of such modes with an emphasis on the chirality and the localization properties of exact zero-modes and possible correlations to topological structures like monopoles.

\section{Formalism}

The Wilson gauge action reads

$$
S[U]=\beta \sum_{x, \mu>\nu}\left(1-\cos \theta_{x, \mu \nu}\right),
$$

where the gauge fields are represented by group elements $U_{x, \mu}=\exp \left(i \theta_{x, \mu}\right) \in$ $U(1)$ with $\theta_{x, \mu} \in(-\pi, \pi]$. The plaquette angles are given by $\theta_{x, \mu \nu}=\theta_{x, \mu}+$ $\theta_{x+\hat{\mu}, \nu}-\theta_{x+\hat{\nu}, \mu}-\theta_{x, \nu} \in(-4 \pi, 4 \pi)$. This compact realization of the gauge fields and the action introduces higher order self-interactions. The pure gauge theory has a confinement phase for small $\beta$ and a Coulomb phase above a phase transition which for this action is close to $\beta \approx 1$ and weakly 1st order [12. 13] (for a recent study of the phase transition suggesting a new order parameter cf. Ref. [14). One observes an abundance of monopoles in the confined phase. Monopoles in compact $U(1)$ are defined following Ref. [15] from deficit angles of the plaquettes bordering 3-cubes, corresponding to links on the dual lattices. Due to current conservation the links form closed loops (better: networks [16]) on the dual lattice. 
The massless overlap operator $D_{\text {ov }}$ may be written

$$
\mathcal{D}_{\mathrm{ov}}=1+\gamma_{5} \epsilon(H)
$$

where $\epsilon$ denotes the operator sign-function and $H=\gamma_{5}\left(s-H_{0}\right)$ is some Hermitian Dirac operator, constructed from an arbitrary Dirac operator. It is convenient to use for $H_{0}$ the usual Wilson Dirac operator with negative mass term and $s$ is a real parameter which can be adjusted such as to optimize the convergence in the construction of $\epsilon(H)$. If $H_{0}$ is already an overlap operator then $\mathcal{D}_{\text {ov }}=H_{0}$ for $s=1$.

For a $U(1)$ gauge theory on manifolds with torus topology there is no topological charge in the sense of a non-vanishing Pontryagin index. So the Atiyah-Singer index theorem may be realized only trivially, i.e. allowing for a cancellation between the numbers of left-handed and right-handed zeromodes. However, on the lattice we have non-differentiable fields and therefore we may expect other violations as well.

It has been demonstrated in QCD that for instanton configurations the Dirac operator shows a zero-mode localized in space-time [7, 17, 18, 8]. To our knowledge nothing is known about the localization properties of zeromodes in QED.

In order to study the localization properties a simple set of gauge invariant quantities has been introduced. For an eigenvector $\psi(x)$ of the lattice Dirac operator one defines a local density

$$
p_{\sigma}(x)=\sum_{d} \psi(x)^{*} \Gamma_{\sigma} \psi(x),
$$

where $\Gamma_{\sigma}$ is an element of the Clifford algebra and the local sum runs over the Dirac indices. The eigenvectors are normalized to unit norm, i.e.

$$
\sum_{x} p_{0}(x)=\left\langle\psi^{\dagger} \psi\right\rangle=1
$$

For the cases of particular interest we abbreviate the scalar density $p(x)=$ $p_{0}(x)$ (for $\Gamma_{0}$ the unit matrix) and the chiral density $p_{5}(x)$ (for $\gamma_{5}$ ). We also determined the vector densities $p_{\mu}$ (for $\Gamma=\gamma_{\mu}$ ), the axial vector densities $p_{5 \mu}\left(\right.$ for $\left.\Gamma=\gamma_{5} \gamma_{\mu}\right)$ and the tensor densities $p_{\mu \nu}\left(\right.$ for $\left.\Gamma=\gamma_{\mu} \gamma_{\nu \neq \mu}\right)$.

Exact zero-modes are also eigenmodes of $\gamma_{5}$ with eigenvalues \pm 1 . Therefore for such eigenmodes we expect even locally

$$
p(x)= \pm p_{5}(x) .
$$


The integral over the chiral density provides a measure for the amount of chiral symmetry breaking because it takes on its largest value for the zeromodes, namely \pm 1 for normalized eigenvectors, and vanishes otherwise.

The so-called inverse participation ratio (IPR) is introduced for further quantification of the localization (see for example Ref. [7]). We define it for both, $p$ and $p_{5}$ :

$$
I=V \sum_{x} p(x)^{2}, \quad I_{5}=V \sum_{x} p_{5}(x)^{2}
$$

where $V$ is the lattice volume.

Assume that the density is evenly distributed on a subvolume $V_{f}$ with $p(x)=1 / V_{f}$ and vanishing elsewhere. Then we find $I=V / V_{f}$, with the limiting cases $I=1$ for distribution over the whole lattice and $I=V$ for localization at one point. It is essentially the inverse fraction of the volume contributing to the mode. This makes it an appropriate measure for the localization of eigenmodes.

Let us try to understand why the inverse participation ratio $I_{5}$ may be considerably smaller than $I$ for near-zero-modes than for exact zero-modes. The local Dirac sum may be written as a sum of two positive terms $p(x)=$ $p_{+}(x)+p_{-}(x)$ where $p_{5}(x)=p_{+}(x)-p_{-}(x)$. Therefore $p(x) \geq\left|p_{5}(x)\right|$. Due to this property $I_{5}$ is bounded for all eigenmodes: $I \geq I_{5}$. For exact eigenmodes we have $I=I_{5}$ due to (6).

Consider now eigenmodes corresponding to eigenvalues far from the origin: $p_{5}(x)$ will be much smaller than $p(x)$ and fluctuate around zero. In this case $I_{5}$ is expected to be significantly smaller than $I$. When the eigenvalues approach zero $I_{5}$ increases and the ratio $I_{5} / I$ is expected to approach 1 .

We also determine different densities $p_{\sigma}$ for exact zero-modes of chirality +1 or -1 . This amounts to checking relations like e.g.

$$
\left\langle\bar{\psi} \mid \gamma_{\mu} \gamma_{5} \psi\right\rangle= \pm\left\langle\bar{\psi} \mid \gamma_{\mu} \psi\right\rangle
$$

which we could verify.

\section{Simulation and results}

The background gauge fields were obtained by using the Metropolis and overrelaxation updating algorithm. All configurations are well decorrelated, separated by 5000 updating sweeps. We analyzed 400 configurations on the $4^{4}$, 

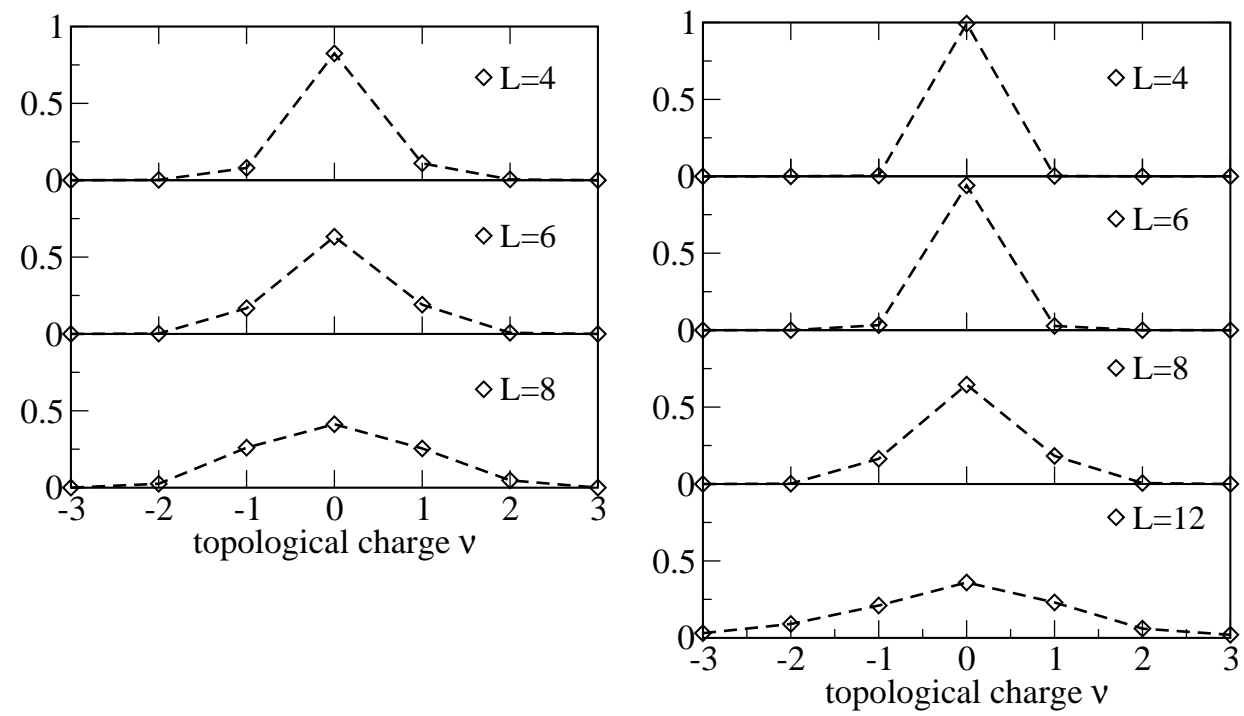

Figure 1: The plots show the normalized histogram (probability distribution) for the topological charge $\nu$ for the configurations at $\beta=0.9$ (left-hand plot) and $\beta=0.99$ (right-hand plot).

$6^{4}$ and $8^{4}$ lattices and 100 on $12^{4}$ lattices. For the computation of the eigenvalues and eigenvectors the so-called implicitly restarted Arnoldi method [19] was used. The overlap operator was computed by an appropriate series expansion. Only the smallest 10 eigenvalues and their eigenvectors were calculated. Real eigenmodes at zero have exact values $\left\langle\bar{\psi} \mid \gamma_{5} \psi\right\rangle= \pm 1$.

In the confined phase many exact zero-modes were observed whereas no zero-modes appeared in the Coulomb phase. Zero modes are chiral eigenmodes and are related to the topological charge via the Atiyah-Singer index theorem. In our discussion we therefore identify the number of zero-modes with positive or negative chirality in a configuration with the positive or negative topological charge $\nu$. We never observed configurations which have zero-modes of different chirality. To our knowledge there is no formal theorem explaining this feature in $4 \mathrm{D}$ (there exists such a vanishing theorem in $2 \mathrm{D}[20]$ ).

In Fig. 1 we show the number $\nu$ of exact zero-modes with chirality \pm 1 for two different values of $\beta$. We observe obvious volume dependence. The number of zero modes as well as the average percentage of zero-mode configu- 


\begin{tabular}{rrrrrrl}
\hline$l$ & $\#_{\text {conf }}$ & $n(\nu=0)$ & $n(\nu=1)$ & $n(\nu=2)$ & $n(\nu=3)$ & $10^{4} \times\left\langle\nu^{2}\right\rangle / V$ \\
\hline 4 & 400 & 397 & 3 & 0 & 0 & $0.29(17)$ \\
6 & 400 & 376 & 24 & 0 & 0 & $0.46(9)$ \\
8 & 500 & 323 & 173 & 4 & 0 & $0.92(6)$ \\
12 & 100 & 36 & 44 & 15 & 5 & $0.72(10)$ \\
\hline
\end{tabular}

Table 1: Summary of configuration number with topological charge for $\beta=$ 0.99 and the corresponding topological susceptibility.

rations compared to all configurations grows with the volume. The integrated number of zero-modes (i.e. adding up all $|\nu|) n_{\text {zeros }} / n_{\text {configs }}$ reaches values $\mathcal{O}(0.9)$ for the large lattices. This can be observed for both $\beta=0.99$ and $\beta=0.9$. On $4^{4}$ lattices at $\beta=0.99$ we found only 3 such modes on all 400 configurations (compared to e.g. 24 zero-modes on $6^{4}$ ). This small number can possibly be attributed to the situation that the pseudocritical point (i.e. the peak position of the specific heat and other cumulants) on smaller lattices effectively occurs already at smaller $\beta$-values and thus we may have been already in or at least closer to the Coulomb phase for that lattice size. For $6^{4}$ the position of the pseudocritical point is near 1.002 whereas for $12^{4}$ it is near 1.010, cf. Ref. [13.

Table 1. summarizes the results for $\beta=0.99$ together with the topological susceptibility per unit volume. Its behavior is compatible with approaching a constant for large volumes.

Following the definitions from Ref.s [15, 21] we determined the monopoles (which are closed loops on the dual lattices). We find no correlation between the monopole density and the number of exact zero-modes on the corresponding configurations. For $\beta=0.9$ the monopole density is $0.19(1)$ for both lattices sizes $6^{4}$ and $8^{4}$, for $\beta=0.99$ it varies from 0.118 to 0.121 for $|\nu|$ ranging from 0 to 3 on the largest lattice size $12^{4}$. For $\beta>\beta_{c}$ the density decreases exponentially. We see no correlation between the number of zeromodes and the integrated monopole loop lengths at $\beta=0.9$ and 0.99 ; this observation confirms [11].

The scaling of the density of small near-zero-modes in the sector of different zero mode number has been compared with Random Matrix Theory expectations in Ref. [11]. The universality class was identified to be the unitary ensemble. We also studied the densities for our data and agree with these findings. Due to our choice of a different offset parameter value $s$ in the Wilson action entering the overlap operator construction, the condensate 

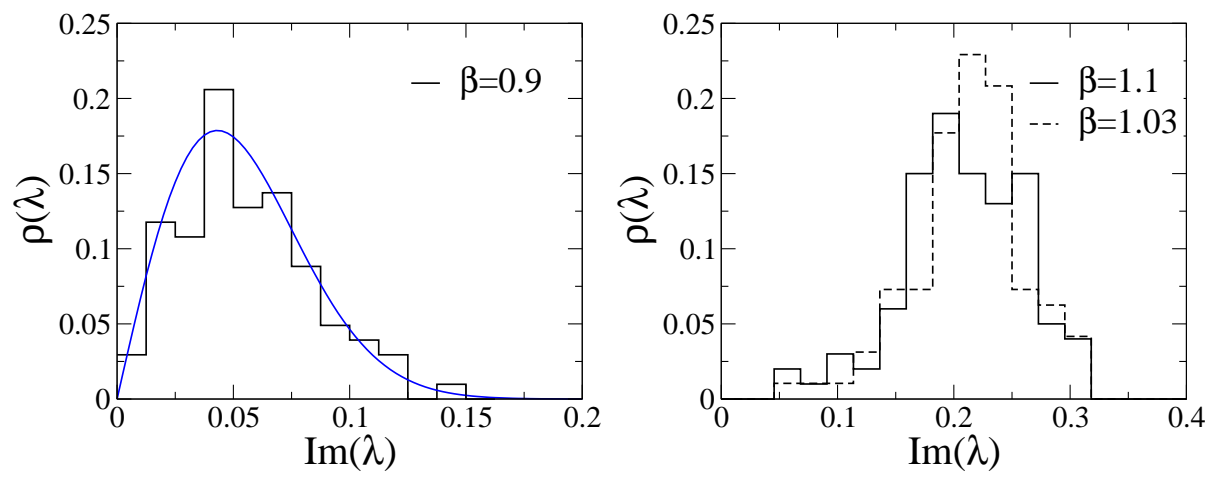

Figure 2: We compare the densities of the smallest eigenvector for $\beta=0.9$ (in the $\nu=0$ sector) and $\beta=1.03$ and 1.1 for lattices size $8^{4}$.

value (and with it the scaling parameter) has to be renormalized multiplicatively 22] for the comparison.

Fig. 2 compares the densities for the confinement region with those for the Coulomb region, exhibiting a different behavior. In the confinement region one expects for $\nu=0$ a distribution following the chiral unitary ensemble of Random Matrix Theory [23] and we overlay a fit to that functional behavior

$$
\rho(z)=\frac{z}{2} \exp \left(-z^{2} / 4\right) .
$$

In the Coulomb region the results for $\beta=1.03$ and $\beta=1.1$ are compatible with each other indicating that there is no gap, different to the observed behavior in the deconfined phase in QCD. Our statistics is not sufficient to exclude a small gap, though.

For the zero-modes we observe $p(x)= \pm p_{5}(x)$ as expected for exact GWfermions and thus also the IPRs agree. For chiral eigenmodes we expect local agreement of those densities which correspond to relations for the gamma matrices like $\gamma_{1} \gamma_{2}=-\gamma_{3} \gamma_{4} \gamma_{5}$, i.e. $p_{12}(x)=-p_{34}(x)$. Indeed the tensor densities for the chiral modes do obey this relation within their group. The local densities for vector and axial vector both vanish. For non-zero-modes these relations are not valid.

In general the integrated tensor densities are comparatively small in absolute magnitude $(0.1 \ldots 0.01)$ for zero-modes. For near-zero-modes all integrated vector, and tensor densities are small $(0.1 \ldots 0.01)$. The integrated pseudoscalar density was exactly \pm 1 as expected for exact zero-modes. 


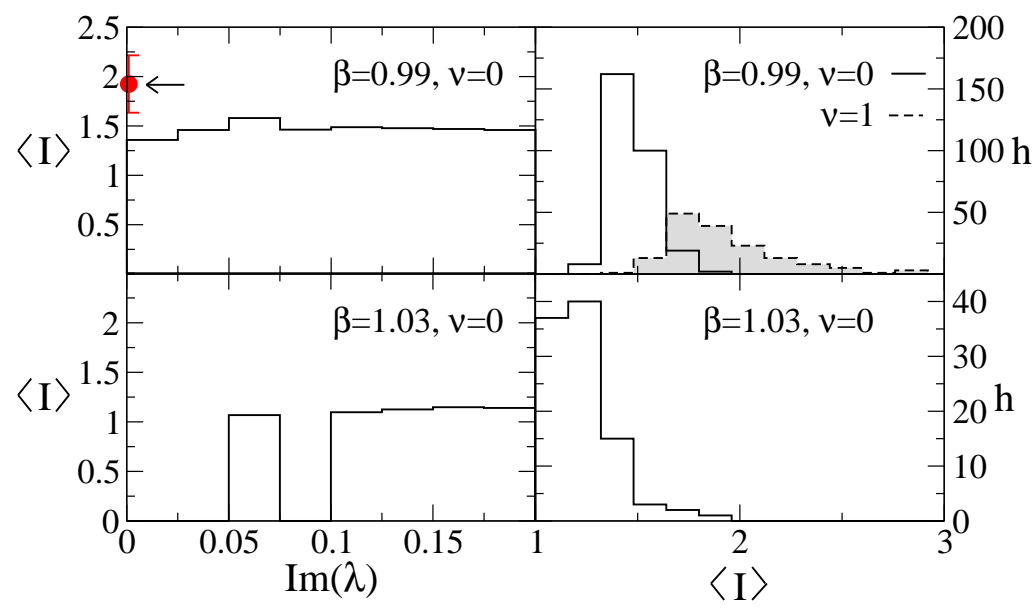

Figure 3: The graphs show the mean inverse participation ratio for the near-zeromodes (in the $\nu=0$ sector) at $\beta=0.99$ and $\beta=1.03$ (lattice size $8^{4}$ ): mean values of IPR (l.h.s.) and IPR-histograms (r.h.s.). The exact zero modes in the $\nu=1$ sector have a wider distribution (shaded area) extending to higher values; the $\langle I\rangle$ and s.d. for those is indicated by the filled circle (arrow) and error bars in the upper l.h.s. plot.

The inverse participation ratio should allow some information on the space-time localization of the eigenmodes. In Fig. 3 we summarize this quantity for the modes for non-zero eigenvalues, binned according to $\operatorname{Im}(\lambda)$ and also show the IPR histograms. In the confinement region we find no increase of the localization for the lowest bins. This observation differs from results in QCD studies [17], where also the near-zero modes show stronger localization.

In the Coulomb region no modes at very small $\operatorname{Im}(\lambda)$ were observed and the low-lying modes show small IPR, close to the minimum of 1 .

Let us now concentrate on the exact zero-modes in the confining phase. These are well localized objects with comparatively large individual IPR values up to 4.2 . For fixed $\beta$ the $\langle I\rangle$ show little if any volume dependence with values of $1.92(29)$ for $L=8$ and 2.03(69) for $L=12$ (this independence on the volume was also observed in recent QCD studies [24]). These values are, however, clearly larger than the mean values for the small non-zero modes. The difference is mainly due to an IPR distribution extending to much higher individual IPR values, as seen in Fig. 3. The values of IPR for the lowest non-zero modes for the sector with no or that with one exact zero 


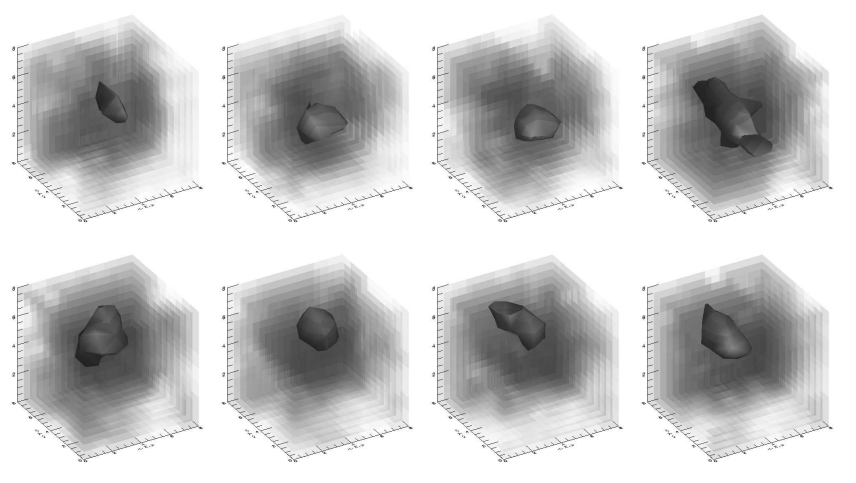

Figure 4: The density shape for zero-mode eigenvector of a Monte Carlo generated gauge configuration (at $\beta=0.99$, lattice size $8^{4}$ ). The $3 \mathrm{D}$ cuts are for time slices $n_{t}=1 \ldots 4$ (first row) and $n_{t}=5 \ldots 8$ (second row). This zero-modes has $I=2.75$.

mode agree within small errors.

In an attempt to visualize the geometrical shape of these localized zeromodes we studied 3D cuts of the density distribution. Fig. 4 gives an example of the observed structure. We plot the surfaces of constant density $p(x)$ for an exact zero-mode for the eight 3D time slices $\left(n_{t}=1 \ldots 8\right)$ of the $8^{4}$ lattice. The shape describes a tubular structure in $4 \mathrm{D}$. However, most of the observed shapes are less clear and often made up from disconnected pieces. Contrary to QCD [18 no clear instanton-like picture (i.e. 4D "blobs") evolves. The structure we observe for the zero-mode eigenvectors are sometimes ball-like in $4 \mathrm{D}$, sometimes tube-like, even tubes closed in some direction due to the periodic boundary conditions. Although a relation to the concept of closed monopole loops (cf. Ref. 21]) may be conjectured, no stringent conclusion can be drawn.

This evidence supports the notion, that the zero-modes are not instantonlike lumps, but extended and periodically closed in one or more directions. In a recent study 24] of the zero-modes in QCD it was argued, that the scaling of $\langle I\rangle$ with the lattice spacing may be used to identify the co-dimension of these objects. Due to lack of results at several different lattice spacing we cannot attempt this here. In Ref. [24] values of $\langle I\rangle \approx 2.8$ were observed, i.e. larger than our values $\langle I\rangle \approx 2$, indicating even stronger localization.

In order to gain further insight, we determined for some gauge config- 

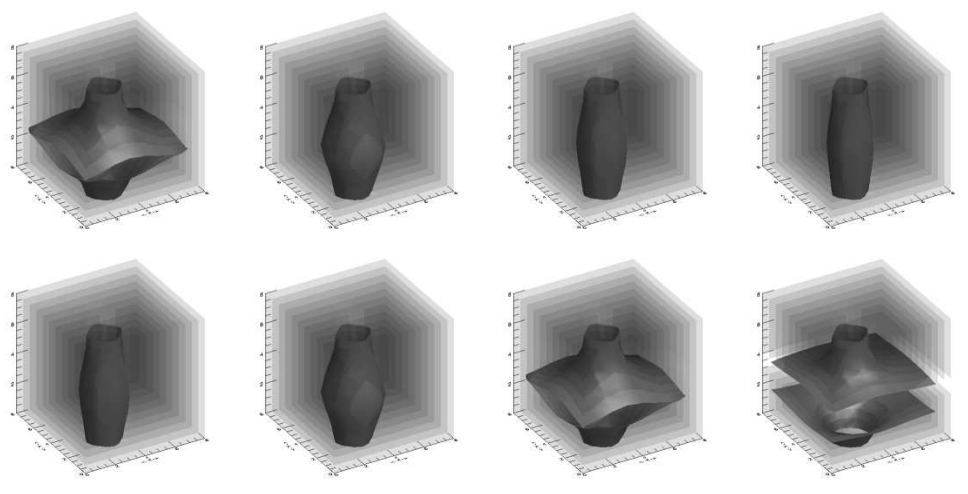

Figure 5: The density shape for zero-mode eigenvector of the constructed gauge configuration (lattice size $8^{4}$ ). The $3 \mathrm{D}$ cuts are for slices $n_{t}=z \ldots 4$ (first row) and $n_{z}=5 \ldots 8$ (second row). The inverse participation ratio for that mode is $I=1.85$.

urations the eigenmodes of the Dirac operator applying also anti-periodic boundary conditions in the time direction by multiplying all temporal gauge links of a timeslice with -1 . This leaves the gauge action invariant (although it is not a gauge transformation on the finite lattice). The number of monopoles and Dirac plaquettes does not change, either.

However, the characteristic polynomial coefficients of the eigenvalue equation for the Dirac matrix involve traces over closed loops and thus may differ due to the periodically closed loops. The Dirac matrix was diagonalized again for the such modified gauge configuration and the eigenvalues were compared with the ones without an added phase: Quite often the zero-modes disappeared. Where the zero-mode survived the transformation, the 3-D density of the new eigenvectors had a completely different structure. This confirms the suspicion that for $U(1)$ gauge theory the zero modes are related to lumps extending in at least one direction. Changing the boundary conditions in QCD sometimes also repositions topological objects (see e.g. [25] and references therein) and in a recent analytical study it was reported [26] that zero modes with constant curvature may be constructed in the $U(1)$ subgroup. These modes may be "switched" on and off by changing the boundary conditions. This is in agreement with our observation.

In $2 \mathrm{D}$ the Pontryagin index is constructed from $\int d \sigma_{\mu \nu} F_{\mu \nu}$. On the lat- 
tice for compact $U(1)$ gauge theory and with torus topology various explicit configurations with such topological charge have been explicitly constructed as well as observed in Monte Carlo simulations (e.g. in Ref. [27]). In 4D the topological charge is proportional to $\int d^{4} x F_{\mu \nu} \tilde{F}_{\mu \nu}$ and a possible method [28, 29] to construct objects with non-vanishing topological charge is e.g. to combine two-dimensional submanifolds, both with topological charge in the $2 \mathrm{D}$ definition. Intersections of $2 \mathrm{D}$ surfaces in $4 \mathrm{D}$ may be points as well as more complicated, e.g. line-like objects. In Ref. [11] such a configuration as suggested in Ref. [29] has been studied and identifies as leading indeed to exact zero-modes. For unit topological charge (lattice size $L^{4}, \omega=2 \pi / L^{2}$ ) it has the form

$$
\begin{aligned}
U_{1}(x)= & \exp \left(i \omega x_{2}\right) \\
U_{2}(x)= & 1 \text { for } x_{2}=1 \ldots L-1, \\
& \exp \left(-i \omega L x_{1}\right) \text { for } x_{2}=L,
\end{aligned}
$$

and for $U_{3}$ and $U_{4}$ equivalent, with $x_{3}$ and $x_{4}$ replacing $x_{1}$ and $x_{2}$. Fig. 5 shows the geometric shape of the eigenvector density of this mode and we observe tubular structures. Note, however, that the tubes are in the 3D intersections, i.e. extend like planes in 4D.

\section{Conclusion}

We have studied zero-mode properties of the overlap Dirac operator for $U(1)$ gauge theory in the confined phase. We find no correlation between the occurrence of these modes and the monopole density confirming [11.

We do observe individual zero-modes with definite chirality. This would contradict the Atiyah-Singer index theorem if applied naively for lattice systems. However, for $U(1)$ lattice gauge theory for compact gauge fields the situation changes. In particular the confinement phase of this system has no analytic continuation to the weak coupling continuum limit. In the confinement phase one can explictly construct configurations with topological charge and zero-modes [29], essentially by combining two 2D sub-manifolds with non-vanishing 1st Chern number.

In the Coulomb phase there are no zero-modes and also the near-zeromodes are suppressed. There we find no pronounced localization signal. In the confinement phase the exact zero-modes are definitely more localized than 
other eigenmodes (as exhibited by the inverse participation ratio). The IPR of the nearby non-zero modes does not seem to depend on the $\operatorname{Im}(\lambda)$. The geometrical structure of the zero eigenmodes has no clear signature singling out 4D blobs - in contrast to e.g. the case of instantons in QCD, which have been observed in distorted hyperspherical shapes. All evidence points to tubular or even planar structures supporting the density function $p(x)$ for a zero-mode.

Acknowledgment: We want to thank Elizabeth Gasparim, Christof Gattringer and Pushan Majumdar for discussion. Support by Fonds zur

Förderung der Wissenschaftlichen Forschung in Österreich, project P P16310N08 is gratefully acknowledged.

\section{References}

[1] M. Atiyah and I. M. Singer, Ann. Math. 93 (1971) 139.

[2] T. Banks and A. Casher, Nucl. Phys. B 169 (1980) 103.

[3] M. Salmhofer and E. Seiler, Commun. Math. Phys. 139 (1991) 395; ibid. 146 (1992) 637.

[4] P. H. Ginsparg and K. G. Wilson, Phys. Rev. D 25 (1982) 2649.

[5] M. Lüscher, Phys. Lett. B 428 (1998) 342.

[6] R. Narayanan and H. Neuberger, Phys. Lett. B 302 (1993) 62; Nucl. Phys. B 443 (1995) 305.

[7] C. Gattringer, M. Göckeler, C. B. Lang, P. E. L. Rakow, and A. Schäfer, Phys. Lett. B 522 (2001) 194.

[8] C. Gattringer, Phys. Rev. Lett. 88 (2002) 221601.

[9] I. Horvath et al., Phys. Rev. D 66 (2002) 034501.

[10] C. Gattringer, Phys. Rev. D 67 (2003) 034507.

[11] B. A. Berg, U. M. Heller, H. Markum, R. Pullirsch, and W. Sakuler, Phys. Lett. B 514 (2001) 97; Nucl. Phys. B (Proc. Suppl.) 106 (2001) 592 , 
[12] I. Campos, A. Cruz, and A. Tarancón, Nucl. Phys. B 528 (1998) 325; Nucl. Phys. B (Proc. Suppl.) 73 (1999) 715.

[13] G. Arnold, T. Lippert, K. Schilling, and T. Neuhaus, Nucl. Phys. B (Proc. Suppl.) 94 (2001) 651.

[14] M. Vettorazzo and Ph. de Forcrand, Nucl. Phys. B 686 (2004) 85.

[15] T. A. DeGrand and D. Toussaint, Phys. Rev. D22 (1980) 2478.

[16] W. Kerler, C. Rebbi, and A. Weber, Phys. Rev. D 50 (1994) 6984.

[17] C. Gattringer, M. Göckeler, P. E. L. Rakow, S. Schäfer, and A. Schäfer, Nucl. Phys. B 618 (2001) 205; Nucl. Phys. B 617 (2001) 101.

[18] C. Gattringer and C. B. Lang, Comput. Phys. Commun. 147 (2002) 398.

[19] D. C. Sorensen, SIAM J. Matrix Anal. Appl. 13 (1992) 357; R. B. Lehoucq, D. C. Sorensen, and C. Yang, (1998).

[20] M. M. Ansourian, Phys. Lett. 70B (1977) 301; J. Kiskis, Phys. Rev. D 15 (1977) 2329; N. K. Nielsen and B. Schroer, Nucl. Phys. B 127 (1977) 493.

[21] V. Grösch et al., Phys. Lett. 162B (1985) 171.

[22] Y. Kikukawa, R. Narayanan, and H. Neuberger, Phys. Rev. D 57 (1998) 1233.

[23] S. M. Nishigaki, P. H. Damgaard, and T. Wettig, Phys. Rev. D 58 (1998) 087704.

[24] C. Aubin et al. [MILC], hep-lat/0410024, 2004.

[25] C. Gattringer and R. Pullirsch, Phys. Rev. D 69 (2004) 094510; C. Gattringer and S. Solbrig, hep-lat/0410040, 2004.

[26] M. Garcia Pérez, Zero modes on constant curvature backgrounds, Talk presented at the workshop The QCD Vacuum fro a Lattice Perspective Univ. Regensburg, Germany, July 29-31, 2004. 
[27] C. R. Gattringer, I. Hip, and C. B. Lang, Phys. Lett. B 409 (1997) 371; C. Gattringer and I. Hip, Nucl. Phys. B 536 (1998) 363.

[28] P. van Baal, Comm. Math. Phys. 85 (1982) 529; ibid. 94 (1984) 397.

[29] J. Smit and J. C. Vink, Nucl. Phys. B 286 (1987) 485. 